\title{
Number of Lymph Nodes Examined
}

National Cancer Institute

\section{Source}

National Cancer Institute. Number of Lymph Nodes Examined. NCI Thesaurus. Code C124446.

The number of lymph nodes that were examined. 